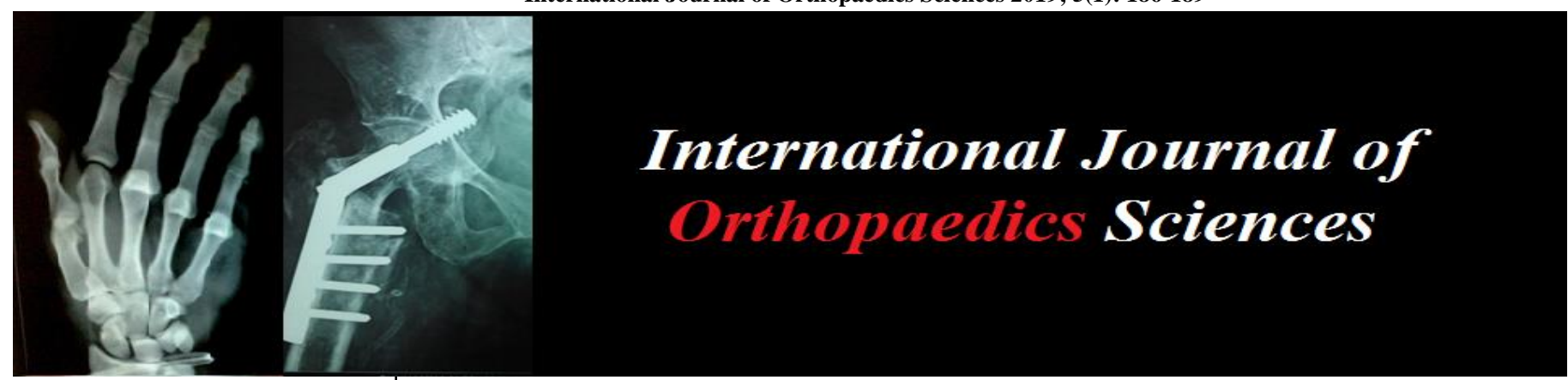

ISSN: $2395-1958$

IJOS 2019; 5(1): 186-189

(C) 2019 IJOS

www.orthopaper.com

Received: 14-11-2018

Accepted: 16-12-2018

\section{Dr. Deviprasad}

Associate Professor, Dept. of Orthopaedics, SRM Medical

College and Research Centre,

Potheri, Chennai,

Tamil Nadu, India

Dr. Suraj Babbar

Associate Professor,

Dept. of Orthopaedics, SRM

Medical College and Research

Centre, Potheri, Chennai,

Tamil Nadu, India
Correspondence

Dr. Suraj Babbar

Associate Professor,

Dept. of Orthopaedics, SRM

Medical College and Research

Centre, Potheri, Chennai,

Tamil Nadu, India

\section{Results of technique of intrafocal pinning in the management of distal radius fractures}

\section{Dr. Deviprasad and Dr. Suraj Babbar}

DOI: https://doi.org/10.22271/ortho.2019.v5.i1d.35

\section{Abstract}

Background and Objectives: Fractures of distal end of the radius are the most common fractures that orthopedic surgeons have to treat. Range of fracture extends from simple extraarticular to highly communited intraarticular fractures. Our objective was to to assess the outcome of intra focal pinning in distal radius fractures in terms of functional recovery and radiological union and to assess the complications associated with the procedure.

Materials and Methods: The present prospective study was conducted between January 2016 and September 2017. 30 patients were enrolled for the study and follow up for a period of 6 months. Closed reduction and percutaneous pinning with $\mathrm{K}$ wire were done. The radiological parameters were assessed for pre-reduction, post reduction, 4 weeks, 3 months and at 6 months. The data from the radiological parameters was analyzed using students t-test, Bonferroni comparison test and chi-square test. The values were normally distributed (normality test $\mathrm{P}=0.3$ ) and significance was assessed using a paired t-test. The statistical significance was defined as $p<0.01$.

Results: Mean age was 42.03 years with 19 male and 11 female patients. $18(60 \%)$ were due to RTA and10 $(33.3 \%)$ were due to fall. Pin tract infection was seen in 2 patients, K-wire loosening was encountered in 1 patient, Loss of reduction in 1 patient, Radiocarpal arthritis in 2 patients. The radiological parameters were assessed for pre-reduction, post reduction, 4weeks and at 3 months. There was a significant difference in all the three parameters i.e. volar tilt, radial length and radial inclination between both the groups at the end of 3 months.

Conclusion: From our study, we conclude that percutaneous pinning technique is a simple, cheap, minimally invasive and effective method for the treatment of unstable extra articular distal radius fractures, with good functional results.

Keywords: Distal radius, percutaneous pinning, closed reduction

\section{Introduction}

Fractures of distal end of the radius are the most common fractures that orthopedic surgeons have to treat. Range of fracture extends from simple extraarticular to highly communited intraarticular fractures. Nearly $16 \%$ of all fractures that are treated in emergency departments involve the distal end of the radius ${ }^{[1]}$. Many fractures of the distal end of radius are in fact relatively uncomplicated and are effectively treated with closed reduction and immobilization in a plaster cast. However, fractures that are either unstable and/or involve the articular surfaces can jeopardize the integrity of the articular congruence and/or the kinematics of these articulations ${ }^{[1]}$. Some of these fractures are caused by severe high energy trauma, resulting in intra-articular involvement and comminution. Treatment of such injuries is difficult. These fractures often are unstable, are difficult to reduce anatomically, and are associated with a high prevalence of complications of post-traumatic osteoarthrosis. Intrafocal pinning is defined as the insertion of pins into the fracture site that can be used to lever the displaced distal fragment into position. Once adequate reduction is achieved, the pins are driven into the metaphysis of the proximal fragment of the radius. The pins act to buttress the distal fragments while maintaining fracture reduction. Distal radius fractures with significant comminution may also require supplemental skeletal stabilization to preserve the length of the radius. Supplemental skeletal stabilization includes external fixation and internal spanning bridge plate techniques ${ }^{[2]}$. Generically the wires function in two ways, either to maintain reduction between two fragments (interfocal pinning) or else to buttress the distal fragments, thereby preventing

$$
\sim 186 \sim
$$


displacement as well as shortening (intrafocal pinning). The basic benefit of this technique is it is more stable fracture than conservative casts. Also preferred in elderly patient with reduced bone quality ${ }^{[3]}$. When the dorsal angle is greater than 20 , radial inclination is below 10 , and radial shortening is more than $6 \mathrm{~mm}$, there are definite functional consequences ${ }^{[4]}$.

\section{Materials and methods}

The present prospective study conducted between January 2016 and September 2017. 30 patients were enrolled for the study and follow up for a period of 6 months. Adults with extra-articular fractures, Unstable fractures, Radiologicalsignificant deformity in the presence of comminution of the dorsal fragment, Failure to maintain reduction of the fracture post reduction while the patient was still under general anesthesia fracture that displaced in cast, were included in the study.

Multiple trauma or other injuries, Open fractures, Neurovascular injuries, associated musculoskeletal injuries to same arm, Patients who lost for follow up, Patients having dementia and psychiatric illness were excluded from the study.

Closed reduction and percutaneous pinning with $\mathrm{K}$ wire are done with patient in supine position. After adequate anesthesia is achieved, the hand was prepared and draped, a closed reduction is performed. Closed reduction is assessed and confirmed under $\mathrm{C}$-arm imaging in both anterior-posterior and lateral planes. A small incision is made at the site of pin insertion, and the tissue protector for the drill is used to prevent injury to the sensory branches of the radial nerve. Kirschner wires are used as the intrafocal pins. The pins required to correct the radial inclination are driven into the radial cortex. The pins are then levered to redirect them proximally before driving them across the opposite cortex in both the posterior-anterior plane and in the lateral plane. Dorsal rotation is corrected. Three or four pins are usually required. Loss of radial inclination and/or radial translation are corrected with one to three Kirschner wires using one pin placed between the first and second compartment, one just dorsal to the second compartment, and one placed in the
Lister's tubercle, taking care to avoid the extensor pollicis longus. The Kirschner wires are cut under the skin. A short arm cast was applied post operatively in each case. The patients were discharged home when their symptoms allowed finger free movements. They were seen at outpatient clinic at 4 weeks, when the cast was changed, to remove the wires and for check radiographs. They were subsequently referred to physiotherapist.

Standard antero-posterior and lateral radiographs of the wrist were taken with forearm in neutral rotation. The volar tilt of the distal radius expressed as number of degrees from neutral position, the radial length and radial inclination were then measured. The radiological parameters were assessed for prereduction, post reduction, 4 weeks, 3 months and at 6 months. The wires and plaster were removed at 4 weeks. The functional outcome was assessed. The range of movement of the wrist and forearm were measured and compared to the normal contralateral side. The data from the radiological parameters was analyzed using students t-test, Bonferroni comparison test and chi-square test. The values were normally distributed (normality test $\mathrm{P}=0.3$ ) and significance was assessed using a paired t-test. The statistical significance was defined as $p<0.01$.

\section{Results and discussion}

The age of the patients ranged from 21 to 67 years with a mean age of 42.03 years. The maximum incidence of the injury was observed during $3^{\text {rd }}$ and $2^{\text {nd }}$ decade of life. Out of 30 patients, 19 patients were male and 11 patients were female. So, the incidence was significantly higher in male. The male and female ratio is 2:1. Right wrist was involved in 23 cases while the left wrist was involved in 07 cases. 18 $(60 \%)$ cases attained fracture due to motor vehicle accident, $10(33.3 \%)$ cases attained fracture due to fall and $2(6.6 \%)$ attained fracture due to other causes. MVA is the most common mode of injury followed by fall with an outstretched hand. Pin tract infection was seen in 2 patients, Kwire loosening was encountered in 1 patient, Loss of reduction in 1 patient, Radiocarpal arthritis in 2 patients.

Table 1: Mean Radiological Measurements: Table 1: Radial Length

\begin{tabular}{|c|c|c|c|}
\hline & Mean Difference (I-J) & Std. error & P-value \\
\hline Post op K- wiring with closed reduction & 1.258 & .299 & HS \\
\hline 4th week K- wiring with closed reduction & 2.288 & .308 & HS \\
\hline 3 months K- wiring with closed reduction & 1.936 & .287 & HS \\
\hline
\end{tabular}

Table 2: Radial Inclination

\begin{tabular}{|c|c|c|c|}
\hline & Mean Difference (I-J) & Std. error & P-value \\
\hline Post op K- wiring with closed reduction & 1.911 & .213 & HS \\
\hline 4th week K- wiring with closed reduction & 3.557 & .185 & HS \\
\hline 3 months K- wiring with closed reduction & 2.991 & .216 & HS \\
\hline
\end{tabular}

Table 3: Volar Tilt

\begin{tabular}{|c|c|c|c|}
\hline & Mean Difference (I-J) & Std. error & P-value \\
\hline Post op K- wiring with closed reduction & -3.616 & .370 & HS \\
\hline 4th week K- wiring with closed reduction & -5.636 & .419 & HS \\
\hline 3 months K- wiring with closed reduction & -5.449 & .414 & HS \\
\hline
\end{tabular}

The radiological parameters were assessed for pre-reduction, post reduction, 4weeks and at 3 months. There was a significant difference in all the three parameters i.e. volar tilt, radial length and radial inclination between both the groups at the end of 3 months.
It has been traditionally taught that fractures of lower end of the radius are more common in postmenopausal females. In young males it is due to high energy trauma and in elderly females it is due to insufficiency fracture. With a greater number of vehicles on road and increased industrialization, 
number of patients sustaining high energy trauma has increased.

While anatomical reduction can usually be achieved by closed manipulation, there is still no agreement as to the most appropriate way of maintaining fractures. Although several studies on the use of percutaneous k-wires for the stabilization of distal radius fractures have been published ${ }^{[5,6-11]}$, their use in elderly remain uncertain as seen by McQueen ${ }^{[12]}$. Studies by Clancey ${ }^{[7]}$, Atkinson ${ }^{[8]}$, Shankar ${ }^{[9]}$ and Walton ${ }^{[13]} 45$ have included a heterogenous group of fractures of different age groups and different regimen of treatment. Although good results have been claimed, controlled trials are lacking. Stoffelen and Broos ${ }^{[56]}$ conducted a prospective randomized trial comparing closed reduction with intrafocal pinning for extra-articular fracture. They found no difference in outcome between groups.

The movements that are present at the wrist are palmar flexion, dorsiflexion, ulnar deviation, radial deviation. The movement that are present at distal radioulnar joint are supination and pronation. Since intraarticular fractures can affect the range of motion at the joint their restoration is important to achieve good results. Physiotherapy and rehabilitation are required and have to be planned carefully. In the present study the average of movements at the affected wrist are palmar flexion $43.7^{0}$, dorsiflexion $54^{0}$, ulnar deviation $21.1^{0}$, radial deviation $13.1^{\circ}$, supination $72.6^{\circ}$ and pronation $71.6^{0}$.

The range of motion is slightly lesser but are comparable to other studies. Supination and pronation seem to be affected less. This probably is due to the result of less education and less vigorous physiotherapy in Indian patients and our series included mostly young adults with high energy trauma and intraarticular involvement.

In any surgical procedure there are chances of complications, fractures of lower end of the radius are not an exception. The complications can be early and late complications. The early complications include loss of reduction, median nerve injury, damage to superficial branch of radial nerve, tendon injuries. The late complications include radiocarpal arthritis, distal radioulnar joint dissociation and arthrosis, loss of reduction and secondary deformity, extensor tendon rupture.

Complications was seen in $6(20 \%)$ patients. In the present series $2(6 \%)$ patient had pin tract infection which was controlled with appropriate antibiotics based on culture and sensitivity report. There was $1(3 \%)$ case of loosening of $\mathrm{K}$ wire. $1(3 \%)$ patient who initially had fixation with $\mathrm{K}$ wire there was loss of reduction and was repeated with external fixation and $\mathrm{K}$ wire augmentation 4 days later. 2(6\%) patients developed radiocarpal arthritis grade 1 as described by Knirk and Jupite ${ }^{[14]}$.

None of the patients had median nerve complications fortunately. The complications in our series is higher than Kapoor series and is lower than Bradway and Jupiter series. Bradway and Jupiter had longer followed up and included late complications which is not seen in our case and hence lower complication rate. Long term follow up is required to assess late complications.

We considered immobilization of fracture for 4 weeks to be adequate and no significant displacement occurred in between. We found statistically significant differences in radiological parameters between the two groups which was similar to the results found by Azzopardi ${ }^{[15]}$. But standardizing lateral views of wrist can be difficult, and the magnitude of difference found were within errors of measurement. In our study we assessed functional outcome by
Quick DASH unlike other studies where Mayo wrist score by Azzopardi ${ }^{[15]}$ and ADL (activities of daily living) by Wong ${ }^{[16]}$ were used. The improvement in functional outcome and range of movement in patients treated by supplementary wires was not statistically significant. This supports opinion of McQueen ${ }^{[12]}$ that k-wires do no gain sufficient purchase in osteopenic bone in elderly patients to maintain anatomical reduction of fracture and to improve function.

\section{Conclusion}

From our study, we conclude that percutaneous pinning technique is a simple, cheap, minimally invasive and effective method for the treatment of unstable extra articular distal radius fractures, with good functional results. It should not be used alone for patients with severe osteopenia, marked dorsal radial comminution, and an associated distal metadiaphyseal ulna fracture and in patients with both volar and dorsal comminution of the distal radius.

\section{References}

1. Ruch David S. Fractures of the distal Radius and Ulna, Chapter 26 in Rockwood and Green's Fractures in Adults, Philadelphia: Lippincott Williams \& Wilkins; $7^{\text {th }} \mathrm{Ed}, 2006,829-831$.

2. Wayne Weil M et al. Treatment of distal radius fractures with intrafocal (Kapandji) pinning and supplemental skeletal stabilization. Hand Clinics. 2005; 21:317-328.

3. Brady $\mathrm{O}$, Rice $\mathrm{J}$ et al. The unstable distal radial fracture one-year post Kapandji intrafocal pinning Injury-Int. Journal of the care of the injured. 1999; 30:251-255.

4. Antonio Neto P, Fabia Lhamby C. Fixation of fractures of the distal extremity of the radius using the modified Kapandji technique: Evaluation of the radiological results Rev Bras Ortop. 2011; 46(4):368-373.

5. Clancey G. Percutaneous Kirschner-wire fixation of Colles fractures: a prospective study of thirty cases. J Bone Joint Surg [Am]. 1984; 66-A:1008-14.

6. Stoffelen DV, Broos PL. Closed reduction versus Kapandji-pinning for extra-articular distal radial fractures. J Hand Surg [Br]. 1999; 24:89-91.

7. Shankar NS, Craxford AD. Comminuted Colles fracture: a prospective trial of management. J R Coll Surg Edin. 1992; 37:199-202.

8. Lenoble E, Dumontier C, Goutallier C, Apoil A. Fracture of the distal radius: a prospective comparison between trans-styloid and Kapandji fixations. J Bone Joint Surg [Br]. 1995; 77-B:562-7.

9. Rayhack JM. The history and evolution of percutaneous pinning of displaced distal radius fractures. Orthop Clin North Am. 1993; 24:287-300.

10. McQueen MM. Redisplaced unstable fractures of the distal radius: a randomized prospective study of bridging versus non-bridging external fixation. J Bone Joint Surg [Br]. 1998; 80-B:665-9.

11. Walton NP, Brammar TJ, Hutchison J, Raj D, Coleman NP. Treatment of unstable distal radial fractures by intrafocal, intramedullary K-wires. Injury. 2001; 32:3839.

12. Stoffelen DV, Broos PL. Closed reduction versus Kapandji-pinning for extra-articular distal radial fractures. J Hand Surg [Br]. 1999; 24:89-91.

13. Shankar NS, Craxford AD. Comminuted Colles' fracture: a prospective trial of management. JR Coll Surg Edin. 1992; 37:199-202.

14. Jupiter JB, Knirk JL. Intra-articular fracture of the distal 
end of the radius. J Bone Joint Surg. 1991; 73-A:461-69.

15. Azzopardi T, Ehrendorfer S, Coulton T, Abela M. A prospective randomized study of immobilization in a cast versus supplementary percutaneous pinning. J Bone Joint Surg Br. 2005; 87-B(6):837.

16. Wong TC, Chiu CY, Tsang WL, Leung WY, Yam SK, Yeung SH. Casting versus percutaneous pinning for extraarticular fractures of the distal radius in an elderly Chinese population. J Hand Surg Eur. 2010; 35(3):202-8. 